\section{Mivacurium, Fixation Error and Human Factors}

\author{
Moiz Alibhai ${ }^{1,2 *}$ and Sarah Wheatly ${ }^{3}$ \\ 'Department of Anaesthesia, Royal Oldham Hospital, Oldham, Lancashire, \\ UK
}

${ }^{2}$ Department of Anaesthesia, North West School of Anaesthesia, Health Education England, Manchester, UK

${ }^{3}$ Department of Anaesthesia, University Hospital of South Manchester, Manchester, UK

\begin{abstract}
An adult male patient in his fifth decade of life presented for elective surgical resection of a long standing neck mass, and underwent general anesthesia with tracheal intubation facilitated by mivacurium neuromuscular blockade. Intraoperative nerve monitoring was utilized by our surgical colleagues to assist in identification of cranial nerves within the surgical field.

Two hours after mivacurium administration, and following tracheal extubation, progressive hypercapnic respiratory failure necessitated repeat tracheal intubation, invasive mechanical ventilation and sedation for further three hours. Post event analysis confirmed atypical plasma cholinesterase phenotype with low plasma cholinesterase concentration, confirming mivacurium sensitivity, also known as mivacurium apnoea.
\end{abstract}

\section{Key Message}

We discuss human factors, miscommunication and misunderstandings that led to the fixation error of incorrectly refuting postoperative residual neuromuscular blockade as being the most probable diagnosis.

Keywords: Fixation error; Human factors; Mivacurium

\section{Introduction}

We acknowledge the widely known subject area of mivacurium sensitivity, but we would like to present this case to highlight human factors that led us to incorrectly believe that spontaneous neuromuscular recovery had already occurred.

There is now a wealth of information in current speciality and non-speciality specific literature that enforces our understanding of the importance of human factors in the outcome of both crisis and non-crisis situations. The subject area of human factors is growing,

*Corresponding author: Moiz Alibhai, Department of Anaesthesia, Royal Oldham Hospital, Oldham, Lancashire, UK, Tel: +44 7974705358; E-mail: moizuk@yahoo.co.uk

Citation: Alibhai M, Wheatly S (2017) Mivacurium, Fixation Error and Human Factors. J Anesth Clin Care 4: 020.

Received: January 16, 2017; Accepted: February 19, 2017; Published: March 06, 2017 and more knowledge and understanding of this area can only help us in becoming more aware of our thinking and behaviour processes.

'Fixation error', 'Confirmation bias', 'Looking for Ghosts' are commonly used terms in the human factors subject area, and we will discuss these terms within the context of our case report.

\section{Case Report}

An adult male patient in his fifth decade of life and of American Society of Anesthesiologists' Physical Status 1 had previously undergone General Anesthesia (GA) for excision of a submandibular gland uneventfully. His preoperative assessment and investigations were all unremarkable. GA was induced and maintained using a propofol and remifentanil Total Intravenous Anesthesia (TIVA) technique. There was a surgical requirement for intraoperative nerve monitoring within the operative area, thus neuromuscular paralysis was achieved with Intravenous (IV) injection of $15 \mathrm{mg}$ of Mivacurium ( $0.2 \mathrm{mg}$ per $\mathrm{kg}$ ).

Tracheal Intubation was performed using direct laryngoscopy, a Grade 1 Cormack and Lehane view of the larynx was recorded. Both induction and maintenance of GA were uneventful and no further neuromuscular blocking drug were administered. A large cyst was excised, and was later histologically reported as benign. Two hours after induction of GA, and following completion of surgery, TIVA was discontinued. Tracheal extubation was performed upon return of consciousness and partial motor response to verbal commands. Neuromuscular monitoring equipment was not available at this time. A short postoperative period of sub-normal respiratory effort was evident. The patient was continuously communicated to and reassured, however no verbalisation from the patient occurred. Supplementary oxygen was utilized and peripheral pulse oximetry remained normal. However, peripheral arterial blood gas analysis revealed a severe respiratory acidaemia. As we were under the impression that the use of intraoperative nerve stimulation and monitoring by our surgical colleagues was functioning prior to emergence from general anesthesia, we initially excluded the possibility of postoperative residual neuromuscular blockade. Our differential diagnoses at this time, included perioperative stroke, and inadvertent intrathecal local anesthesia administration.

It was only when the lead surgeon communicated with us, that a correct understanding of events occurred. Use of intraoperative nerve stimulation for assisting in identifying relevant cranial nerves had been attempted by our surgical colleagues, but no positive nerve stimulation had occurred. The surgical team had assumed this to be due to technical failure, and continued surgery without informing the rest of the theatre team of the lack of motor response to intraoperative nerve monitoring. On disclosure of this critically important information, neuromuscular monitoring was utilized immediately, and revealed a high degree of neuromuscular blockade. Train of four monitoring revealed a 1 of 4 responses. A total of $5 \mathrm{mg}$ of neostigmine and $1 \mathrm{mg}$ of glycopyrrolate was administered intravenously over two doses. A transient improvement in neuromuscular function was evident, but sub-optimal respiratory effort continued. GA was re-induced with intravenous propofol and midazolam, and tracheal intubation 
performed uneventfully. GA was maintained with a propofol intravenous infusion technique.

A provisional diagnosis of mivacurium sensitivity/apnoea was made, and a peripheral venous blood sample was collected for plasma cholinesterase level analysis. Three hours after repeat induction of GA, and more than five hours after the initial dose of mivacurium, there was qualitative evidence of return of neuromuscular power. No further anticholinesterase drug was administered. Tracheal extubation was uneventful. The patient vocalised immediately and displayed good neuromuscular power.

The patient was discharged to a ward area following a full explanation of events, and has been followed up by the surgical and anesthesia teams. He gives a well articulated verbal account of explicit recall with poor neuromuscular power consistent with residual neuromuscular paralysis.

\section{Discussion and Conclusion}

We would like to take this opportunity to reinforce the clear and ubiquitous recommendation of the use of neuromuscular monitoring throughout the entire perioperative period for those patients who receive neuromuscular paralytic drugs of any kind. A set of recommendations published by a world respected authority in anesthesia states that the use of neuromuscular monitoring, along with other standards, will lead to a reduction in adverse events and improve patient safety. Furthermore, they clarify that the use of neuromuscular monitoring in our profession is still too low, and remind us that clinical assessment alone of the offset of neuromuscular paralytic drugs is inadequate [1]. We believe perioperative neuromuscular monitoring to be mandatory for those patients who receive neuromuscular paralytic drugs.

Post event analysis confirms an atypical plasma cholinesterase phenotype with a low plasma cholinesterase concentration, with low dibucaine, fluoride and scoline numbers. Due to the patient's family circumstances, he is not able to give any family history. Following further analysis, the anesthesia technique for the previous surgical procedure did not utilize suxamethonium or mivacurium.

This case report highlights the consequences of incorrect assumption of functioning intraoperative nerve stimulation and monitoring as part of surgical exposure and dissection of the neck that led to an incorrect assumption of spontaneous recovery of neuromuscular function. The importance of communication between our surgical and anesthesia teams regarding intraoperative nerve monitoring has been emphasized for the benefit of all team members, and it was a lack of this specific communication that led us to initially discount postoperative residual neuromuscular blockade as a cause of the patient's abnormal recovery. Furthermore, we are recognizant of our initial fixation error as senior team members that led to other team members more junior than ourselves into our fixated thinking model. A fixation error is an error that leads us incorrectly down a blind path towards finding a solution to both high and low acuity problems. 'Fixation occurs when anesthetists concentrate on a single aspect of the case to the detriment of other more relevant aspects. Ironically, these are relatively easy to detect only in hindsight' [2]. Our assumption that spontaneous recovery of neuromuscular function had occurred was based on a false premise that our surgical colleagues were achieving positive motor responses to their intraoperative nerve stimulation and monitoring.

A 'confirmation bias' also exists in that we searched for the clinical feature of non vocalization that reinforced one of our alternative diagnosis of perioperative stroke. Other authors talk of what is called a 'perception bias' that exists in the way clinicians incorrectly perceive provided information that can lead human error [2].

Perhaps, a way out of this thinking model could have been to start 'Looking for Ghosts'. A term used to describe an active search for information that needs to be positively identified in order to support the initial diagnosis. In the event of this information not being found, it should automatically trigger a thought process that begins a process of self questioning and respectful questioning from other team members. This is intrinsically linked to maintaining situational awareness.

In a specific paper discussing the clinical applications of human factors in anesthesia, the terms 'fixation errors' and 'insight problems' are related to the 'cheap necklace' problem. This paper also discusses the 'hill climbing heuristic' in which a 'step forward' may actually be a 'step backwards' to find the correct solution to a perceived and/or actual problem [3].

The hill climbing heuristic helps us understand how easy it is to feel we are moving forward to a potential solution, when actually taking a 'step backwards' by pausing to reassess, rethink and taking a 'side step' would be the best step forward to finding the correct solution to the problem. This 'backward step' requires an active conscious decision that will allow us to take a fresh, new approach to solve a problem. We direct readers to some excellent online resources [4-6].

\section{Competing Interests}

'No external funding and no competing interests declared'.

\section{References}

1. Checketts MR, Jenkins B, Pandit JJ (2017) Implications of the 2015 AAGBI recommendations for standards of monitoring during anaesthesia and recovery. Anaesthesia 1: 3-6.

2. Kumar A, Gupta K, Gupta M, Bhandari S (2014) Fatal drug errors in anaesthesia: Can we override? Indian J Anaesth 58:785-786.

3. Fioratou E, Flin R, Glavin R (2010) No simple fix for fixation errors: cognitive processes and their clinical applications. Anaesthesia 65: 61-69.

4. Oxenham S (2016) How fixation error prevents you from solving problems. Big Think, New York, USA.

5. http://chfg.org/about-us/

6. LITFL (2016) Crisis Resource Management (CRM). Life In the fast Lane (LIT$\mathrm{FL})$, Medical Blog. 